\title{
Galhas de insetos em uma área de transição caatinga-cerrado no Nordeste do Brasil
}

\author{
${\text { Elaine Cotrim } \text { Costa }^{1 *} \text {, Sheila Patrícia Carvalho-Fernandes }}^{2, a}$, Juliana Santos-Silva ${ }^{1,3, b}$ \\ ${ }^{1}$ Programa de Pós-Graduação em Biodiversidade Vegetal, Universidade do Estado da Bahia - Campus VIII, \\ Paulo Afonso, Bahia, Brasil. \\ ${ }^{2}$ Programa de Pós-Graduação em Zoologia, Museu de Zoologia, Universidade Federal do Rio de Janeiro, Rio de \\ Janeiro, Rio de Janeiro, Brasil. \\ ${ }^{3}$ Universidade do Estado da Bahia - Campus VI, Caetité, Bahia, Brasil.
}

\begin{abstract}
Resumo - Neste estudo, foi investigado um ambiente de transição caatinga-cerrado no município de Caetité, estado da Bahia, com o objetivo de contribuir para o conhecimento das plantas hospedeiras e insetos galhadores. Foram encontrados 43 morfotipos de galhas, em 17 famílias botânicas. Leguminosae e Myrtaceae apresentaram maior riqueza de galhas, com 15 e cinco morfotipos, respectivamente. As galhas estavam presentes principalmente em folhas (70\%), e foram predominantemente globoides (58\%), isoladas $(67,44 \%)$, uniloculares $(82,60 \%)$ e verdes (51\%). Cecidomyiidae (Diptera) foi a principal família gallhadora. A fauna associada às galhas foi composta por parasitoides, inquilinos, sucessores e predadores. Registra-se pela primeira vez pseudoescorpiões em galhas de Myrcia tomentosa.
\end{abstract}

Palavras-chaves adicionais: insetos galhadores, interação inseto-planta, plantas hospedeiras, pseudoescorpiões, Semiárido.

\begin{abstract}
Insect galls in a caatinga-cerrado transition area in Northeast Brazil) - The caatinga-cerrado transition environments in Caetité, state of Bahia, Brazil, were investigated in order to contribute to the knowledge of the host plants and galling insects. Forty-three gall morphotypes were found, in 17 plant families. Leguminosae and Myrtaceae were the families with the greatest richness of galls, with 15 and five morphotypes, respectively. Galls occurred mainly on leaves (70\%), and were predominantly globoid (58\%), isolated (67.44\%), unilocular $(82.6 \%)$ and green (51\%). Cecidomyiidae (Diptera) was the main gall inducing family. The fauna associated with the galls comprised parasitoids, inquilines, successors and predators. We recorded for the first time pseudoscorpions in galls of Myrcia tomentosa.

Additional key words: galling insects, insect-plant interaction, host plants, pseudoscorpion, semiarid region.
\end{abstract}

Galhas são alterações estruturais e anatômicas do tecido vegetal em resposta à ação de organismos indutores como ácaros e insetos, culminando na formação de uma estrutura muitas vezes simétrica (Raman 2007). A maioria dos insetos galhadores mantém uma relação espécie-específica com as plantas hospedeiras e está geralmente restrita a determinados órgãos (Floate et al. 1996). A galha é considerada o fenótipo estendido do inseto galhador e confere a este abrigo, alimento e proteção contra inimigos naturais (Stone \& Schönrogge 2003).

Apesar do aumento do número de estudos de galhas nos ecossistemas brasileiros, ainda são necessários estudos que contribuam para o conhecimento da fauna de galhadores. Alguns destes ecossistemas foram amostrados apenas recentemente, enquanto outros permanecem à espera de investigação. No Brasil, os levantamentos de galhas estão concentrados nas Regiões Sul e Sudeste, principalmente em ambientes de cerrado (Gonçalves-Alvim \& Fernandes 2001; Coelho et al. 2009), restinga (Maia 2001; Maia 2013a) e floresta atlântica (Fernandes et al. 2001; Fernandes \& Negreiros 2006). Na Região Nordeste, os poucos

\footnotetext{
*Autora para correspondência: elainecostabio@gmail.com;

asheilapcfernandes@gmail.com; bjullybandeira@hotmail.com

Editor responsável: Alessandro Rapini

Submetido: 27 set. 2014; aceito: 6 dez. 2014

Publicação electrônica: 15 dez. 2014; versão final: 20 jan. 2015
}

estudos concentram-se em Pernambuco (Santos et al. 2009, 2011, 2012) e na Bahia, Sergipe e Alagoas (Carvalho-Fernandes et al. 2012; Costa et al. 2014), onde o conhecimento da diversidade de galhas ainda é escasso.

O Semiárido brasileiro compreende todos os estados da Região Nordeste do Brasil, além do norte de Minas Gerais. Por apresentar características peculiares, como altas temperaturas e escassez de chuvas, essa região é rica em endemismos, com animais e plantas adaptados a condições extremas. Entretanto, pouco se conhece sobre sua biodiversidade. Isto é preocupante, pois o Semiárido vem sendo bastante modificado pelo desmatamento, queimadas e culturas irrigadas (Leal et al. 2003).

A nascente do riacho Jatobá, uma Área de Preservação Permanente (APP) próxima ao perímetro urbano do município de Caetité, na região da Serra Geral do estado da Bahia, se insere neste contexto. Seu entorno apresenta uma grande diversidade florística, devido às fitofisionomias ali representadas (caatinga, cerrado, mata de galeria e áreas de transição caatingacerrado). Desse modo, nosso estudo teve como objetivo inventariar e caracterizar as galhas de insetos e as plantas hospedeiras na vegetação de transição entre caatinga e cerrado da APP do riacho Jatobá, ampliando o levantamento prévio de insetos galhadores em espécies de Leguminosae dessa área (Costa et al. 2014). 


\section{MATERIAIS E MÉtodos}

Área de estudo. A nascente do riacho Jatobá pertence à bacia hidrográfica do Rio de Contas e à subbacia do rio São João. O ponto de afloramento fica aproximadamente a $1,5 \mathrm{~km}$ do centro de Caetité $\left(14^{\circ} 04^{\prime} 36,8^{\prime \prime} \mathrm{S}, 42^{\circ} 29^{\prime} 59,7^{\prime \prime} \mathrm{W}\right)$, cerca de 974 m s.n.m. O clima é seco, subúmido a semiárido, com temperatura média anual de $20,7^{\circ} \mathrm{C}$ (IBGE 2012), estação chuvosa de novembro a janeiro e pluviosidade média de 841 mm (CEI 1994). A vegetação do entorno da nascente é composta por áreas de caatinga, cerrado, mata de galeria e áreas de transição caatinga-cerrado.

Etapa de Campo. As coletas foram concentradas nas bordas das trilhas de acesso à nascente na APP, adentrando a vegetação em até $1 \mathrm{~m}$. Foram realizadas 10 coletas, de $3 \mathrm{~h}$ cada, mensalmente, no período de julho de 2012 a abril de 2013. As plantas hospedeiras de galhas com mais de $1 \mathrm{~m}$ de altura foram marcadas e georreferenciadas com aparelho GPS (modelo Garmim Vista HCX) para posterior observação, e ramos, preferencialmente férteis, foram coletados e prensados. As galhas foram fotografadas, descritas e classificadas de acordo com as suas características morfológicas externas (e.g., órgão de ocorrência, cor, forma, pubescência e agrupamento), adotando a terminologia proposta por Isaias et al. (2013). O material coletado foi armazenado separadamente em sacos plásticos e levado ao Laboratório de Botânica da Universidade do Estado da Bahia.

Etapa de Laboratório. Os ramos das plantas hospedeiras coletados foram herborizados e depositados no Herbário da Universidade do Estado da Bahia (HUNEB), coleção de Caetité. O material botânico foi identificado com base em literatura especializada, na análise de fotografias da coleção-tipo e na consulta a especialistas. A listagem das plantas foi organizada seguindo o APG III (2009).

As galhas foram armazenadas em potes plásticos forrados com papel de filtro para emergência dos indutores e fauna associada. Algumas galhas foram dissecadas sob estereomicroscópio para obtenção das fases imaturas e observação do número de câmaras. Os insetos (indutores e fauna associada) foram preservados em álcool 70\% e identificados com auxílio de um microscópio estereoscópio.

\section{RESUltados}

Foram encontrados 43 morfotipos de galhas entomógenas, em 33 espécies de plantas hospedeiras pertencentes a 17 famílias e 28 gêneros (Tabela 1; Figuras 1-3). Leguminosae e Myrtaceae apresentaram as maiores riquezas tanto de galhas ( 15 e 5 morfotipos, respectivamente), como de espécies galhadas ( 9 e 4 espécies, respectivamente). O gênero com maior riqueza de galhas foi Bauhinia L. (LeguminosaeCaesalpinoideae), com cinco morfotipos.

As galhas foram induzidas em folhas (70\%), caules (21\%), gemas $(7 \%)$ e pecíolo (2\%), sendo verde $(51 \%)$ e marrom $(28 \%)$ as cores mais frequentes. As galhas foram, em sua maioria, globoides (58\%) ou fusiformes $(14 \%)$ e ocorreram isoladamente, sem a formação de aglomerados $(67 \%)$. Com relação à ornamentação das galhas, metade dos morfotipos possuíam tricomas e $82 \%$ apresentaram apenas uma câmara larval.

A ocorrência de galhas em Guatteria odontopetala (Annonaceae), Rollinia leptopetala (Annonaceae), Terminalia fagifolia (Combretaceae), Croton triangularis (Euphorbiaceae), Trichilia emarginata (Meliaceae), Eugenia pitanga (Myrtaceae), Ouratea parviflora (Ochnaceae), Psychotria carrascoana (Rubiaceae), Serjania paludosa (Sapindaceae) e Callisthene microphylla (Vochysiaceae) é assinalada pela primeira vez para o Brasil (Tabela 1).

Das seis ordens conhecidas de insetos galhadores (Diptera, Lepidoptera, Coleoptera, Thysanoptera, Hemiptera e Hymenoptera), três foram registradas neste estudo, Diptera $(n=15)$, Lepidoptera $(n=4)$ e Thysanoptera $(\mathrm{n}=1)$. Devido à escassez do material e ao grande número de parasitoides, 23 indutores não puderam ser identificados $(53,48 \%)$. Dos identificados, a maioria $(75 \%)$ pertence à família Cecidomiiydae (Diptera). A fauna de artrópodes associada às galhas foi observada em 12 morfotipos (Tabela 1), sendo composta por parasitoides da ordem Hymenoptera (Eulophidae, Eurytomidae e Torymidae), inquilinos (Tanaostigmatidae, Hemiptera, Coleoptera e Thysanoptera), sucessores (Collembola, Formicidae) e predadores (Pseudoescorpiones).

\section{DISCUSSÃo}

Nos inventários de galhas realizados no Nordeste brasileiro (Santos et al. 2009, 2011; Silva et al. 2011; Carvalho-Fernandes et al. 2012; Santos et al. 2012; Costa et al. 2014) e em áreas de transição caatingacerrado no Norte de Minas Gerais (Luz et al. 2012), como em outros ecossistemas brasileiros, Leguminosae e Myrtaceae estão entre as famílias com maior riqueza de galhas entomógenas e de espécies galhadas (Maia \& Fernandes 2004; Coelho et al. 2009; Maia 2013a,b). Além disso, Leguminosae é uma família com boa representação na composição florística do Semiárido brasileiro (Queiroz et al. 2006), corroborando a hipótese de que a composição florística local tem grande influência na riqueza de insetos galhadores, como observado em campo rupestre (Coelho et al. 2009) e em floresta atlântica (Maia 2001; Maia 2013a,b). O gênero Bauhinia foi também observado como super-hospedeiro de galhas em um inventário na Serra do Cipó, Minas Gerais, em áreas de cerrado e campo rupestre (Coelho et al. 2009) e em áreas de caatinga em Pernambuco (Santos et al. 2011). 
Tabela 1. Plantas hospedeiras, descrição dos morfotipos, insetos indutores e fauna associada das galhas encontradas em área de transição caatinga-cerrado no munícipio de Caetité, estado da Bahia, Brasil.

\begin{tabular}{|c|c|c|c|c|c|c|c|c|c|c|c|}
\hline \multicolumn{2}{|c|}{ Planta hospedeira } & \multirow{2}{*}{$\begin{array}{l}\text { Órgão } \\
\text { atacado }\end{array}$} & \multirow[t]{2}{*}{ Face } & \multirow[t]{2}{*}{ Forma } & \multirow[t]{2}{*}{ Cor } & \multirow[t]{2}{*}{ Pilosa } & \multirow[t]{2}{*}{ Ocorrência } & \multirow{2}{*}{$\begin{array}{l}\text { Número de } \\
\text { câmara }\end{array}$} & \multirow[t]{2}{*}{ Inseto indutor } & \multirow[t]{2}{*}{ Fauna associada } & \multirow[t]{2}{*}{ Figuras } \\
\hline Família & Espécie & & & & & & & & & & \\
\hline \multirow[t]{4}{*}{ Annonaceae } & $\begin{array}{l}\text { Duguetia furfuracea (A.St.-Hil.) } \\
\text { Saff. }\end{array}$ & Folha & Adaxial & Globoide & $\begin{array}{l}\text { Verde/ } \\
\text { Marrom }\end{array}$ & Sim & Agrupada & 1 & Indeterminado & $\begin{array}{l}\text { Eulophidae } \\
\text { Hymenoptera }\end{array}$ & $1 \mathrm{~A}, \mathrm{~B}$ \\
\hline & Guatteria odontopetala Mart.* & Folha & Abaxial & Globoide & Verde & Não & Simples & 1 & Indeterminado & & $1 \mathrm{C}$ \\
\hline & Rollinia leptopetala R.E.Fr.* & Folha & Adaxial & Globoide & Verde & Sim & Simples & 1 & Indeterminado & & $1 \mathrm{D}$ \\
\hline & Xylopia aromatica (Lam.) Mart. & Gema & - & Globoide & Verde & Sim & Simples & Várias & Lepidoptera & & $1 \mathrm{E}$ \\
\hline Boraginaceae & Cordia sp. & Folha & Abaxial & Globoide & Verde & Sim & Agrupada & 1 & Cecidomyiidae & & $1 \mathrm{~F}$ \\
\hline \multirow[t]{3}{*}{ Combretaceae } & Combretum leprosum Mart. & Folha & Adaxial & Globoide & Vermelho & Não & Simples & 1 & Cecidomyiidae & & $1 \mathrm{G}$ \\
\hline & Terminalia fagifolia Mart.* & Folha & Abaxial & Globoide & Verde & Sim & Simples & 1 & Indeterminado & & $1 \mathrm{H}$ \\
\hline & & Folha & Ápice & Margem enrolada & Verde & Sim & Simples & 1 & Indeterminado & & $1 \mathrm{I}$ \\
\hline Euphorbiaceae & Croton triangularis Müll. Arg.* & Folha & Adaxial & Cônica & Verde & Sim & Simples & 1 & Lepidoptera & & $1 \mathrm{~J}$ \\
\hline \multirow{9}{*}{$\begin{array}{l}\text { Leguminosae - } \\
\text { Caesalpinioideae }\end{array}$} & Bauhinia brevipes Vogel & Folha & Adaxial & Globoide & Marrom & Sim & Agrupada & 1 & Schizomyia & & $1 \mathrm{~K}$ \\
\hline & & & & & & & & & macrocapillata & & \\
\hline & & & & & & & & & $\begin{array}{l}\text { Maia, } 2005 \\
\text { (Cecidomyiidae) }\end{array}$ & & \\
\hline & & Folha & Adaxial & Globoide & Verde & Não & Simples & 1 & Lepidoptera & Hymenoptera & $1 \mathrm{~L}$ \\
\hline & Bauhinia pulchella Benth. & Folha & Adaxial & Globoide & Verde & Não & Simples & 1 & Cecidomyiidae & & $1 \mathrm{M}$ \\
\hline & & Caule & - & Fusiforme & Marrom & Não & Agrupada & Várias & Indeterminado & & $1 \mathrm{~N}$ \\
\hline & & Folha & Adaxial & Cônica & Verde & Não & Simples & 1 & Indeterminado & & $10, \mathrm{P}$ \\
\hline & Copaifera langsdorffii Desf. & Folha & Adaxial & Lenticular & Verde & Não & Simples & 1 & Cecidomyiidae & Thysanoptera & $1 \mathrm{Q}$ \\
\hline & & & Abaxial & Roseta & Marrom & & & & & & $1 \mathrm{R}$ \\
\hline \multirow{7}{*}{$\begin{array}{l}\text { Leguminosae - } \\
\text { Mimosoideae }\end{array}$} & Calliandra sp. & Pecíolo & - & Globoide & Rosa & Sim & Simples & Várias & Indeterminado & Eurytoma sp. & $1 \mathrm{~S}$ \\
\hline & & & & & & & & & & Eurytomidae & \\
\hline & & & & & & & & & & $\begin{array}{l}\text { Tanaostigmatidae- } \\
\text { Hymenoptera }\end{array}$ & \\
\hline & Mimosa gemmulata Barneby & Folha & Adaxial & Globoide & Marrom/ & Sim & Simples/agrupada & 1 & Cecidomyiidae & Collembola, & $1 \mathrm{~T}$ \\
\hline & & & & & verde & & & & & $\begin{array}{l}\text { Thysanoptera, } \\
\text { Hymenoptera }\end{array}$ & $2 \mathrm{~A}$ \\
\hline & & Folha & Adaxial & Globoide & Preto & Não & Simples & 1 & Cecidomyiidae & & $2 \mathrm{~B}$ \\
\hline & & Caule & - & Fusiforme & Marrom & Não & Simples & Várias & Indeterminado & & $2 \mathrm{C}$ \\
\hline
\end{tabular}


E. C. Costa et al. - Galhas em área de transição caatinga-cerrado

\begin{tabular}{|c|c|c|c|c|c|c|c|c|c|c|c|}
\hline \multicolumn{2}{|l|}{ Planta hospedeira } & \multirow{2}{*}{$\begin{array}{l}\text { Órgão } \\
\text { atacado }\end{array}$} & \multirow[t]{2}{*}{ Face } & \multirow[t]{2}{*}{ Forma } & \multirow[t]{2}{*}{ Cor } & \multirow[t]{2}{*}{ Pilosa } & \multirow[t]{2}{*}{ Ocorrência } & \multirow{2}{*}{$\begin{array}{l}\text { Número de } \\
\text { câmara }\end{array}$} & \multirow[t]{2}{*}{ Inseto indutor } & \multirow[t]{2}{*}{ Fauna associada } & \multirow[t]{2}{*}{ Figuras } \\
\hline Família & Espécie & & & & & & & & & & \\
\hline & $\begin{array}{l}\text { Pseudopiptadenia brenanii } \\
\text { G.P.Lewis \& M.P.Lima }\end{array}$ & Caule & - & Globoide & Marrom & Não & Simples & & Indeterminado & & $2 \mathrm{D}$ \\
\hline & $\begin{array}{l}\text { Senegalia langsdorffii (Benth.) } \\
\text { Seigler \& Ebinger }\end{array}$ & Caule & - & Globoide & Marrom & Não & Simples & Várias & Indeterminado & & $2 \mathrm{E}$ \\
\hline & Senegalia sp. & Folha & Adaxial & Lenticular & Verde & Sim & Simples & 1 & Cecidomyiidae & $\begin{array}{l}\text { Hemiptera, } \\
\text { Hymenoptera }\end{array}$ & $2 \mathrm{~F}$ \\
\hline & & Caule & - & Globoide & Marrom & Não & Simples & Várias & Indeterminado & & $2 \mathrm{G}$ \\
\hline $\begin{array}{l}\text { Leguminosae - } \\
\text { Papilionoideae }\end{array}$ & Dalbergia miscolobium Benth. & Folha & Abaxial & Globoide & Verde & Sim & Simples & 1 & Cecidomyiidae & & $2 \mathrm{H}$ \\
\hline \multirow[t]{2}{*}{ Malpighiaceae } & Banisteriopsis sp. & Folha & Abaxial & Cônica & Verde & Não & Simples & 1 & Cecidomyiidae & & $2 \mathrm{I}$ \\
\hline & & Gema & - & Fusiforme & Marrom & Não & Simples & 1 & Cecidomyiidae & & $2 \mathrm{~J}$ \\
\hline Malvaceae & Malvaceae sp. & Folha & Adaxial & Globoide & Verde & Sim & Agrupada & 1 & Indeterminado & $\begin{array}{l}\text { Coccinelidae- } \\
\text { Coleoptera }\end{array}$ & $2 \mathrm{~K}$ \\
\hline \multirow[t]{2}{*}{ Meliaceae } & Trichilia emarginata (Turcz.) & Folha & Adaxial & Globoide & Verde & Não & Simples & 1 & Indeterminado & & $2 \mathrm{~L}$ \\
\hline & C.DC.* & Caule & - & Cônica & Marrom & Não & Agrupada & 1 & Indeterminado & & $2 \mathrm{M}$ \\
\hline \multirow[t]{5}{*}{ Myrtaceae } & Eugenia pitanga (O.Berg) Nied.* & Folha & Adaxial & Lenticular & Verde & Não & Agrupada & 1 & Cecidomyiidae & & $2 \mathrm{~N}$ \\
\hline & Eugenia punicifolia (Kunth) DC. & Caule & - & Fusiforme & Marrom & Não & Agrupada & Várias & Indeterminado & & 20 \\
\hline & Myrcia splendens (Sw.) DC. & Folha & Limbo & Globoide & Verde & Não & Agrupada & Várias & Indeterminado & $\begin{array}{l}\text { Torymidae- } \\
\text { Hymenoptera }\end{array}$ & $2 \mathrm{P}$ \\
\hline & Myrcia tomentosa (Aubl.) DC. & Folha & Borda & Globoide & Vermelho & Sim & Agrupada & 1 & Thysanoptera & & $2 \mathrm{Q}$ \\
\hline & & Caule & - & Fusiforme & Marrom & Sim & Simples & 1 & Indeterminado & $\begin{array}{l}\text { Formicidae- } \\
\text { Hymenoptera, } \\
\text { Pseudoescorpiones }\end{array}$ & $2 \mathrm{R}$ \\
\hline Ochnaceae & Ouratea parviflora Engl.* & Folha & Abaxial & Cônica & Verde & Não & Agrupada & 1 & Indeterminado & & $2 \mathrm{~S}$ \\
\hline Rubiaceae & $\begin{array}{l}\text { Psychotria carrascoana Delprete } \\
\quad \text { \& E.B.Souza* }\end{array}$ & Gema & - & Roseta & $\begin{array}{l}\text { Verde/ } \\
\text { marrom }\end{array}$ & Não & Simples & 1 & Lepidoptera & $\begin{array}{l}\text { Eurytoma sp. } \\
\text { (Eurytomidae, } \\
\text { Hymenoptera) }\end{array}$ & $\begin{array}{l}2 \mathrm{~T} \\
3 \mathrm{~A}\end{array}$ \\
\hline \multirow[t]{2}{*}{ Sapindaceae } & Serjania paludosa Cambess.* & Caule & - & Fusiforme & Marrom & Sim & Simples & 1 & Indeterminado & & $3 \mathrm{~B}$ \\
\hline & Serjania sp. & Folha & Adaxial & Globoide & Verde & Não & Agrupada & 1 & Indeterminado & & $3 \mathrm{C}$ \\
\hline Sapotaceae & Pouteria torta (Mart.) Radlk. & Folha & Adaxial & Lenticular & Marrom & Sim & Simples & 1 & Indeterminado & & $3 \mathrm{D}$ \\
\hline Simplocaceae & Symplocos sp. & Folha & Limbo & Margem enrolada & Verde & Não & Simples & 1 & Indeterminado & & $3 \mathrm{E}$ \\
\hline Trigoniaceae & Trigonia nivea Cambess. & Folha & Abaxial & Globoide & Marrom & Sim & Simples & 1 & Cecidomyiidae & & $3 \mathrm{~F}$ \\
\hline Verbenaceae & Lantana sp. & Folha & Adaxial & Globoide & Verde & Sim & Simples & 1 & Cecidomyiidae & Hymenoptera & $3 \mathrm{G}$ \\
\hline Vochysiaceae & Callisthene microphylla Warm.* & Folha & Adaxial & Globoide & Verde & Não & Simples & 1 & Cecidomyiidae & & $3 \mathrm{H}$ \\
\hline
\end{tabular}



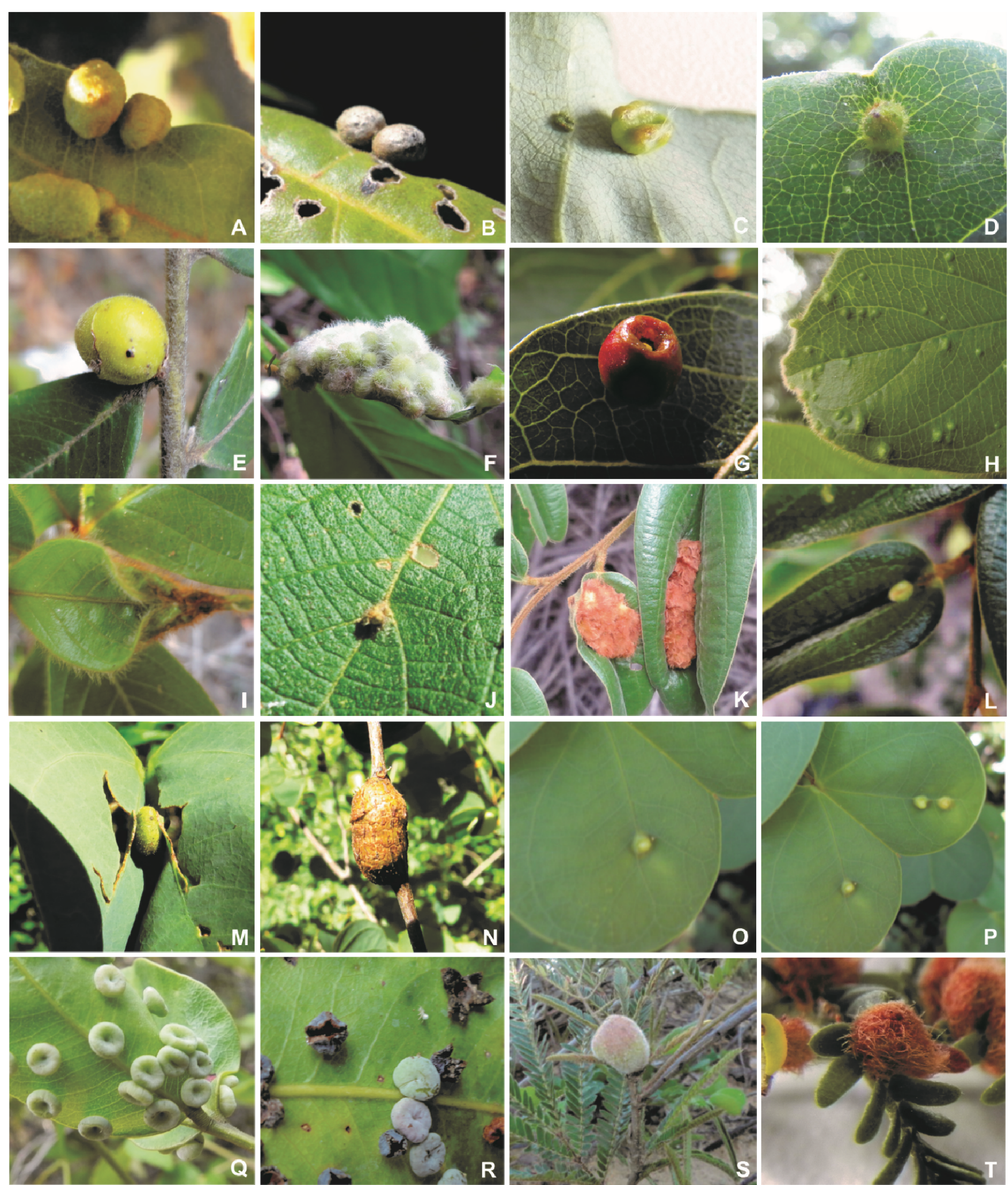

Figura 1. Galhas de insetos em área de transição caatinga-cerrado, Caetité, estado da Bahia, Brasil: A, B- Duguetia fufuracea, galha foliar globoide; C- Guatteria odontopetala, galha foliar globoide; D- Rollinia leptopetala, galha foliar globoide; E- Xylopia aromatica, galha globoide em gema; F- Cordia sp., galha foliar globoide; G- Combretum leprosum, galha foliar globoide; H, I- Terminalia fagifolia, galha foliar globoide (H) e galha de enrolamento da margem foliar (I); J- Croton triangulares, galha foliar cônica; K, L- Bauhinia brevipes, galha foliar globoide (K) e galha foliar globoide (L); M-P- Bauhinia pulchella, galha foliar globoide (M), galha caulinar fusiforme (N) galha foliar cônica (O e P); Q, R- Copaifera langsdorffii, galha foliar lenticular ou roseta; S- Calliandra sp., galha peciolar globoide. T- Mimosa gemmulata, galha foliar globoide. 

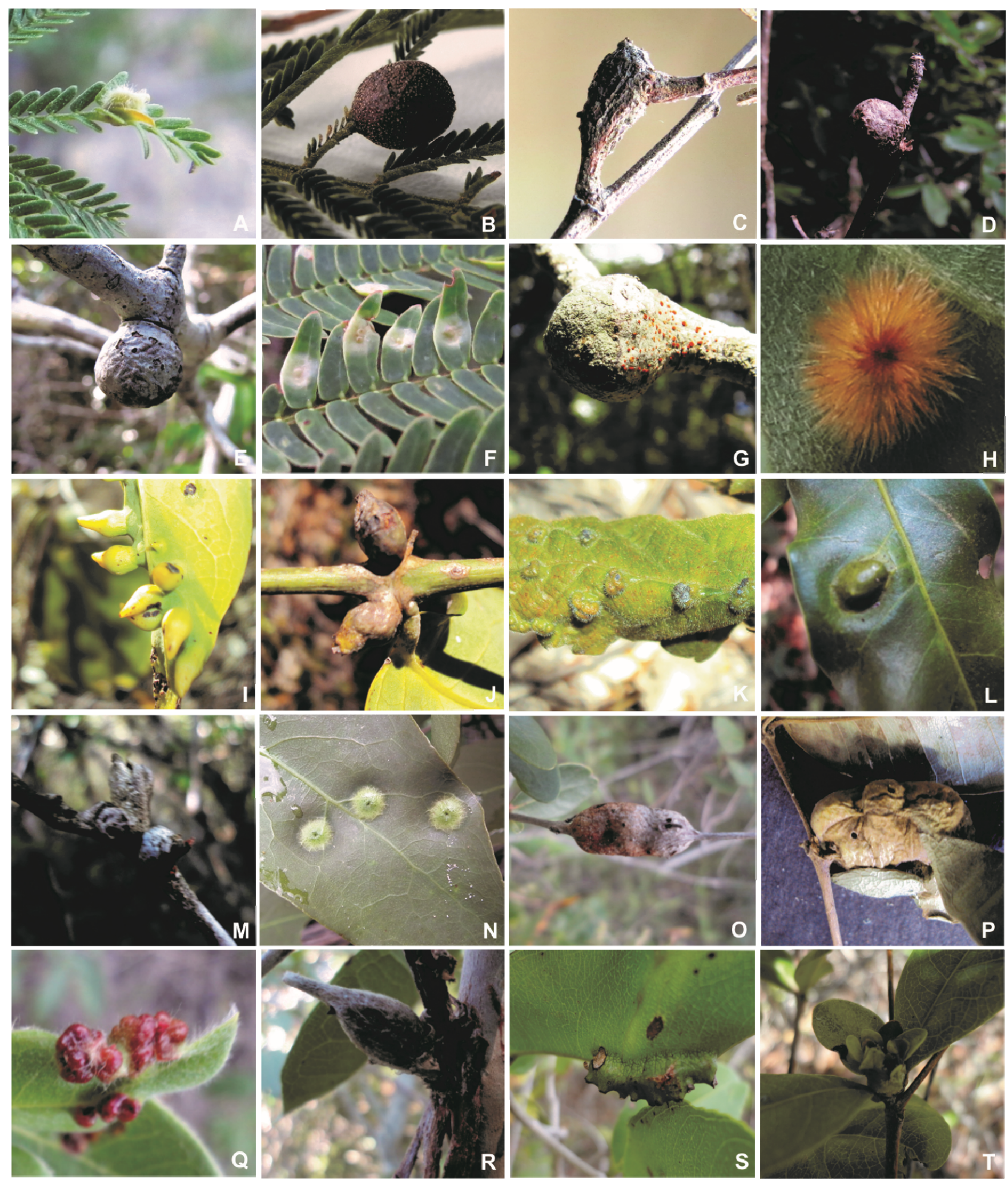

Figura 2. Galhas de insetos em área de transição caatinga-cerrado, Caetité, estado da Bahia, Brasil: A-C- Mimosa gemmulata, galhas foliares globoides (A e B) e galha caulinar fusiforme (C); D- Pseudopiptadenia brenanni, galha caulinar globoide; E- Senegalia langsdorffii, galha caulinar globoide; F, G- Senegalia sp., galha foliar lenticular (F) e galha caulinar globoide (G); H- Dalbergia miscolobium, galha foliar globoide; I, J- Banisteriopsis sp., galha foliar cônica (I) e galha fusiforme em gema (J); K- Malvaceae sp., galha foliar globoide; L, MTrichilia emarginata, galha foliar globoide (L) e galha caulinar cônica (M); N- Eugenia pitanga, galha foliar lenticular; O- Eugenia punicifolia, galha caulinar fusiforme; P- Myrcia splendens, galha foliar globoide; Q, R. Myrcia tomentosa, galha foliar globoide (Q) e galha caulinar fusiforme (R); S- Ouratea parviflora, galha foliar cônica; T- Psychotria carrascoana, galha de roseta em gema. 

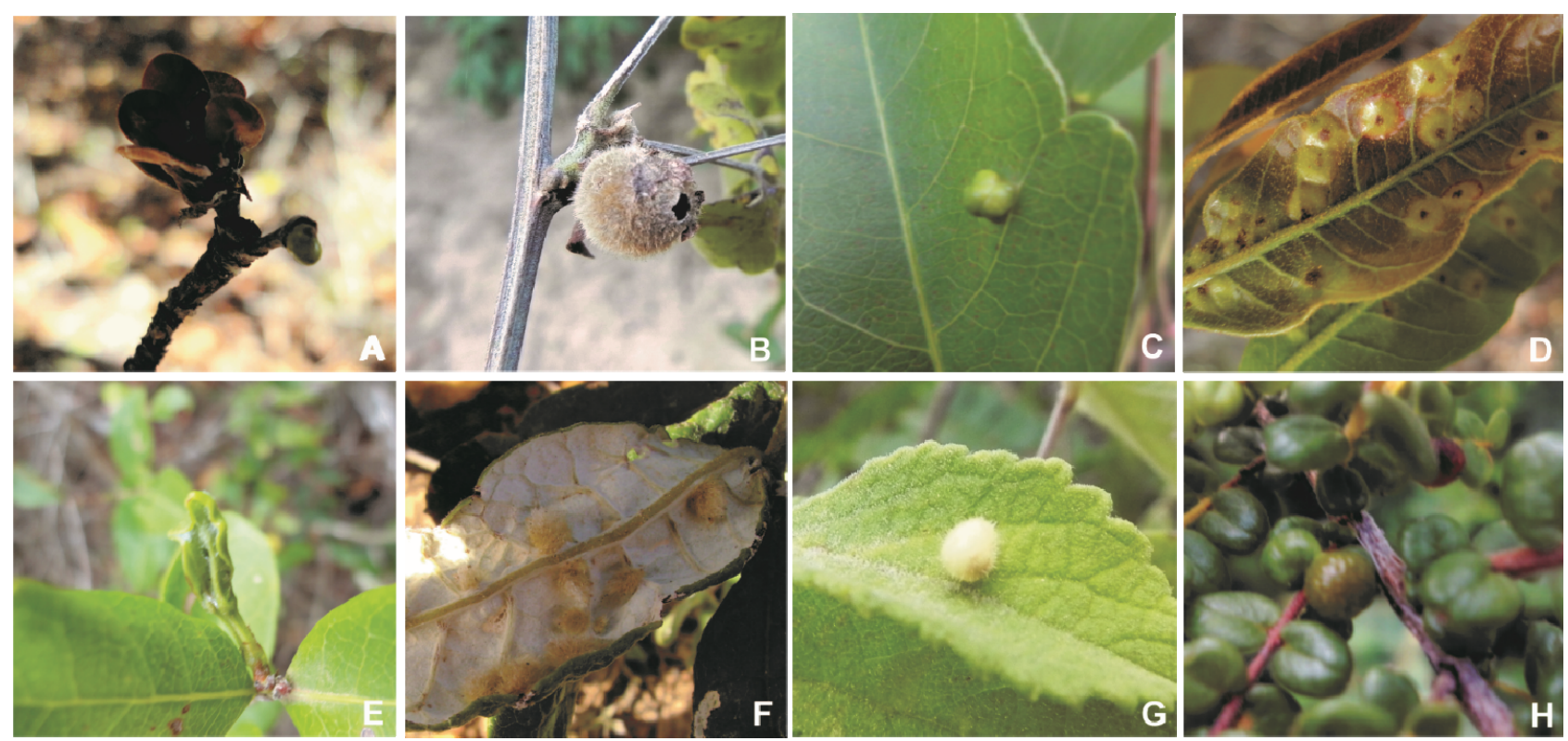

Figura 3. Galhas de insetos em área de transição caatinga-cerrado, Caetité, estado da Bahia, Brasil: A- Psychotria carrascoana, galha de roseta em gema; B- Serjania paludosa, galha caulinar fusiforme; C- Serjania sp., galha foliar globoide; D- Pouteria torta, galha foliar lenticular; E- Simplocus sp., galha de enrolamento da margem foliar. F- Trigonia nivea, galha foliar globoide; G- Lantana sp., galha foliar globoide; H-Callisthene microphylla, galha foliar globoide.

As galhas foram induzidas preferencialmente em folhas, seguidas do caule. Estudos desenvolvidos em diferentes ecossistemas brasileiros (Maia \& Fernandes 2004; Santos et al. 2011; Luz et al. 2012; Maia 2013a) apresentaram resultados semelhantes. Com relação à morfologia externa, a coloração verde das galhas, predominante neste estudo, está relacionada provavelmente à presença de clorofila, o que sugere que essas estruturas também participem da produção de fotoassimilados através da fotossíntese (Dias et al. 2013). Algumas galhas sofrem alterações na coloração ao longo do seu desenvolvimento, como nas galhas foliares de Mimosa gemmulata (Figuras 1T, 2A) e nas galhas de gemas em Psychotria carrascoana (Figuras 2T, 3A). Essas modificações estão provavelmente relacionadas ao desenvolvimento do inseto galhador ou à ação de outros níveis tróficos, como parasitoides (Dias et al. 2013). Como a morfologia das galhas pode representar a diversidade dos insetos galhadores, o acompanhamento mensal nos levantamentos e o cuidado na descrição de tais características é fundamental.

A maioria das galhas apresentou as formas globoide ou fusiforme, que também predominaram em outros inventários no Brasil (Santos et al. 2009; Maia \& Souza 2013), sendo o morfotipo fusiforme mais característico de galhas caulinares (Gonçalves-Alvim \& Fernandes 2001). Metade das galhas apresentaram tricomas; no Brasil, apenas Urso-Guimarães et al. (2003) citaram o predomínio de galhas pubescentes. Os tricomas evitam a perda excessiva de umidade nas câmaras e, consequentemente, ajudam a manter a temperatura interna da galha. Além disso, são estruturas que possivelmente ajudam na proteção contra inimigos naturais (Stone \& Schonrögge 2003).
Galhas isoladas e uniloculares foram frequentes, fato também relatado em áreas na caatinga de Pernambuco (Santos et al. 2011) e de transição no norte de Minas Gerais (Luz et al. 2012).

A principal família de insetos galhadores neste estudo foi Cecidomiiydae (Diptera), concordando com outros levantamentos no Brasil (e.g., Fernandes et al. 2006; Coelho et al. 2009; Maia \& Souza 2013). Esta família vem sendo apontada como principal indutora de galhas na região Neotropical (Gagné \& Jaschhof 2014). As galhas induzidas por Cecidomiiydae estavam associadas principalmente às folhas de Leguminosae. Resultado semelhante foi encontrado em restingas da Região Sudeste do Brasil, onde foi observada uma forte preferência de Cecidomiiydae por folhas, principalmente em espécies de Myrtaceae, Asteraceae e Leguminosae (Maia 2013a).

A microfauna associada a galhas foi composta de parasitoides, inquilinos, sucessores e predadores. A presença de parasitoides é uma das dificultadades na obtenção dos insetos galhadores (Maia \& Souza 2013). Dentre estes, espécies de Eulophidae e Eurytomidae se destacam como os principais inimigos naturais dos galhadores na região neotropical (Maia 2001; Maia \& Fernandes 2004; Maia \& Azevedo 2009). A ocorrência de pseudoescorpiões em galhas é pouco frequente, sendo registrada até o momento apenas para galhas induzidas em Eugenia adstringens Cambess (Myrtaceae), Combretum leprosum (Combretaceae), Handroanthus sp. (Bignoniaceae) e Calophyllum brasiliense Cambess (Calophyllaceae) (Maia 2001, 2002, 2013b; Maia \& Souza 2013). Neste trabalho, a presença de pseudoescorpião é registrada pela primeira vez em galhas de Myrcia tomentosa (Myrtaceae). 
Este levantamento contribui significativamente para o conhecimento da diversidade de insetos galhadores e suas plantas hospedeiras em área de transição caatingacerrado do Nordeste brasileiro. Entretanto, a extensão territorial das áreas de transição na Região é grande e a ampliação deste conhecimento exigirá investigações como esta em outras áreas do Nordeste.

\section{AgRAdeCIMENTOS}

À Fundação de Amparo à Pesquisa do Estado da Bahia (FAPESB) pela concessão da bolsa de iniciação científica a primeira autora (FAPESB 2223/2012), aos pesquisadores Dr. Luciano Paganucci de Queiroz (Universidade Estadual de Feira de Santana) e Dr. Marcos Sobral (Universidade Federal de São João DelRei) pela identificação do material botânico e das Myrtaceae, respectivamente. E a dois revisores anônimos pelas valorosas sugestões.

\section{REFERÊNCIAS}

APG III 2009. An update of the Angiosperm Phylogeny Group classification for the orders and families of flowering plants: APG III. Botanical Journal of the Linnean Society 161: 105-121.

Carvalho-Fernandes, S.P.; Almeida-Cortez, J.S. \& Ferreira, A.L.N. 2012. Riqueza de galhas entomógenas em áreas antropizadas e preservadas de Caatinga. Revista Árvore 36: 269-277.

CEI (Centro de Estatística e Informações) 1994. Informações Básicas de Municípios Baianos: Região Serra Geral. Salvador, Bahia.

Coelho, M.S.; Almada, E.D.; Fernandes, G.W.; Carneiro, M.A.A.; Santos, R.M.; Quintino, A.V. \& Sanchez-Azofeifa, A. 2009. Gall inducing arthropods from a seasonally dry tropical forest in Serra do Cipó, Brazil. Revista Brasileira de Entomologia 53: 404-414.

Costa, E.C.; Fernandes, S.P.C. \& Silva, J.S. 2014. Galhas entomógenas associadas à Leguminosae do entorno do riacho Jatobá, Caeitité, Bahia, Brasil. Revista Brasileira de Biociências 12: $115-120$.

Dias, G.G.; Moreira, G.R.P.; Ferreira, B.G. \& Isaias, R.M.S. 2013. Why do the galls induced by Calophya duvauae Scott on Schinus polygamus (Cav.) Cabrera (Anacardiaceae) change colors? Biochemical Systematics and Ecology 48: 111-122.

Fernandes, G.W. \& Negreiros, D. 2006. A comunidade de insetos galhadores da RPPN Fazenda Bulcão, Aimorés, Minas Gerais, Brasil. Lundiana 7: 111-120.

Fernandes, G.W.; Julião, G.R.; Araújo, R.C.; Araújo, S.C.; Lombardi, J.A.; Negreiros, D. \& Carneiro, M.A.A. 2001 Distribution and morphology of insect galls of the Rio Doce Valley, Brazil. Naturalia 26: 211-244.

Floate, K.D.; Fernandes, G.W. \& Nilsson, J.A. 1996. Distinguishing intrapopulacional categories of plants by their insect faunas: galls on rabbitbrush. Oecologia 105: 21-229.

Gagné, R. J. \& Jaschhof, M. 2014. In: A Catalog of the Cecidomyiidae (Diptera) of the World. Disponível em http://www.ars.usda.gov/SP2UserFiles/Place/80420580/Gagne_
2014_World_Cecidomyiidae_Catalog_3rd_Edition.pdf; acesso em 15 out. 2014.

Gonçalves-Alvim, S.J. \& Fernandes, G.W. 2001. Comunidades de insetos galhadores (Insecta) em diferentes fisionomias do cerrado em Minas Gerais, Brasil. Revista Brasileira de Zoologia 18: 289-305.

IBGE (Instituto Brasileiro de Geografia e Estatística) 2012. Caetité - BA/ Histórico. Disponível em http://www.ibge.gov.br/cidadesat/ painel/painel.php?codmun=290520\#; acesso em 15 fev. 2012.

Isaias, R.M.S.; Carneiro, R.G.S.; Oliveira, D.C. \& Santos, J.C. 2013. Illustrated and annotated checklist of Brazilian gall morphotypes. Neotropical Entomology 42: 230-239.

Leal, I.R.; Tabareli, M. \& Silva, J.M.C. 2003. Ecologia e conservação da Caatinga: Uma introdução ao desafio. In: I.R. Leal, M. Tabareli \& J.M.C. Silva (eds), Ecologia $e$ Conservação da Caatinga. Universidade Federal de Pernambuco, Recife, p. 13-16.

Luz, G.R.; Fernandes, G.W.; Silva, J.O.; Neves, F.S. \& Fagundes, M. 2012. Galhas de insetos em habitats xérico e mésico em região de transição Cerrado-Caatinga no norte de Minas Gerais, Brasil. Neotropical Biology and Conservation 7: 171-186.

Maia, V.C. 2001. The gall midges (Diptera, Cecidomyiidae) from three restingas of Rio de Janeiro State, Brazil. Revista Brasileira de Zoologia 18: 583-629.

Maia, V.C. 2002. Description of the larva of Houardodiplosis rochae Tavares, 1925 (Diptera, Cecidomyiidae, Clinodiplosini) and new record of pseudoscorpions in galls. Revista Brasileira de Entomologia 46: 81-82.

Maia, V.C. 2013a. Insect galls from restingas of Southeastern Brazil, with new records. Biota Neotropica 13: 184-209.

Maia, V.C. 2013b. Insect galls of São Tomé das Letras (MG, Brazil). Biota Neotropica 13: 1-26.

Maia, V.C. \& Fernandes, G.W. 2004. Insect galls from Serra de São José (Tiradentes, MG, Brazil). Brazilian Journal of Biology 64: 423-445.

Maia, V.C. \& Azevedo, M.A.P. 2009. Micro-himenópteros associados com galhas de Cecidomyiidae (Diptera) em Restingas do Estado do Rio de Janeiro (Brasil). Biota Neotropica 2: 1-14.

Maia, V.C. \& Souza, M.C. 2013. Insect galls of the xeric vegetation of Ilha do Cabo Frio (Arraial do Cabo, RJ, Brazil). Biota Neotropica 13: 1-11.

Queiroz, L.P.; Conceição, A. \& Giulietti, A.M. 2006. Nordeste Semi-árido: caracterização geral e lista das fanerógamas. In: A.M. Giulietti \& L.P. Queiroz (orgs), Instituto do Milênio do Semi-árido: diversidade e caracterização das fanerógamas do Semi-árido brasileiro. Vol. 1. Associação Plantas do Nordeste, Recife, p. 15-364.

Raman, A. 2007. Insect-induced plant galls of India: unresolved questions. Current Science 92: 748-757.

Santos, J.C.; Almeida-Cortez, J.S. \& Fernandes, G.W. 2009. Diversity of gall-inducing insects in the high altitude wetland forests in Pernambuco, Northeastern Brazil. Brazilian Journal of Biology 71: 47-56.

Santos, J.C.; Almeida-Cortez, J.S. \& Fernandes, G.W. 2011. Richness of gall-inducing insects in the tropical dry forest (caatinga) of Pernambuco. Revista Brasileira de Entomologia 55: 45-54. 
Santos, J.C.; Almeida-Cortez, J.S. \& Fernandes, G.W. 2012. Gall-inducing insects from Atlantic forest of Pernambuco, northeastern Brazil. Biota Neotropica 12: 197-213.

Silva, P.S.D.; Knoechelmann, C.M.; Tabarelli, M. \& AlmeidaCortez, J.S. 2011. Richness of gall morphospecies along a secondary successional gradient of Atlantic Forest in northeastern Brazil. Revista Brasileira de Biociências 9: 270-277.
Stone, G. N. \& Schönrogge, K. 2003. The adaptive significance of insect gall morphology. Trends in Ecology and Evolution 18: 512-522.

Urso-Guimarães, M.V.; Scareli-Santos, C. \& Bonifácio-Silva, A.C. 2003. Occurrence and characterization of entomogen galls from natural vegetation areas in Delfinópolis, MG, Brazil. Brazilian Journal of Biology 63: 705-715. 